\title{
A study on a new antineural antibody in a case of paraneoplastic sensory neuropathy associated with breast carcinoma
}

\author{
Teruaki Iwahashi, Atsushi Inoue, Chang-Sung Koh, Nobuo Yanagisawa
}

\begin{abstract}
Paraneoplastic sensory neuropathy is a remote effect of cancer, usually associated with small cell lung carcinoma and anti-Hu antibody. This report details the case of a 59 year old woman with a breast carcinoma and a paraneoplastic sensory neuropathy characterised by chronic asymmetric sensory neuropathy. Anti-Hu antibody was not detected in her serum; nor were other known antineuronal antibodies such as anti-Ri and Yo. However, we have found an antineural antibody that reacted to a $106 \mathrm{kDa}$ mouse neural antigen which has not yet been reported. Immunohistochemically, this antineural antibody bound to the posterior grey horn. This finding suggests that this antineural antibody may play an important part in the pathogenesis of the sensory neuropathy of this patient.
\end{abstract}

(F Neurol Neurosurg Psychiatry 1997;63:516-519)

Keywords: paraneoplastic sensory neuropathy; breast cancer; antineural antibody

Neurological paraneoplastic syndrome is a remote effect of carcinoma and some other types of neoplasia on the central or peripheral nervous system. Paraneoplastic sensory neuropathy is one of the neurological paraneoplastic syndromes, characterised by a subacute clinical course of progressive, usually severe, sensory ataxia that often precedes or closely follows the diagnosis of cancer. ${ }^{1-3}$ Although the pathogenesis of neurological paraneoplastic syndromes is not known, recent discoveries of several types of antibodies to neural tissue components in patients with paraneoplastic syndromes, such as paraneoplastic sensory neuropathy, paraneoplastic cerebellar degeneration, paraneoplastic encephalomyelitis, opsoclonus-myoclonus syndrome, and Lambert-Eaton myasthenia syndrome have led to speculation that an autoimmune mechanism for neurological paraneoplastic syndromes may exist. Especially in paraneoplastic sensory neuropathy and paraneoplastic encephalomyelitis, the most common cancer is small cell lung carcinoma and it is associated with anti-Hu antibody. ${ }^{4-8}$ At high titres, it is a sensitive and specific marker for paraneoplastic sensory neuropathy and paraneoplastic encephalomyelitis with small cell lung cancer. We describe a patient with sensory neuropathy and intraductal spreading breast cancer. We detected an IgG antibody in her serum which reacted with mouse neural tissue. This seems to be a new antibody which is different from the known antineural antibodies, and is important in the pathogenesis of sensory neuropathy.

\section{Case report}

A 59 year old woman became aware of numbness in her left arm in 1991. After one month, she noticed difficulty in moving the left arm. In 1992, numbness and dysaesthesia developed in her left leg. In October 1993, she began to have difficulty ascending and descending stairs. In January, 1994, she noticed numbness in her right hand fingers. She was admitted to Shinshu University hospital on 6 October 1994.

On admission, routine physical examination was unremarkable with the exception of a left axillary lymphadenopathy. Neurological examination disclosed pseudoathetosis in her left hand fingers. Muscle strength was almost normal, but movements of the left hand were ataxic.

Tendon reflexes were absent in the limbs. Babinski's sign was elicited bilaterally. Pinprick and touch sensation were decreased in the areas described by the patient as numb. Vibratory sensation was decreased in her limbs and position sensation was lost in her left handfingers.

Laboratory findings on admission were as follows. No abnormal values were found in blood and chemistry tests. A test for M protein was negative. The examination of autoantibodies as a screening for autoimmune diseases was normal with all tumour markers within the normal range. Examination of CSF showed 1 lymphocyte $/ \mathrm{mm}^{3}$ and a total protein value of 43 $\mathrm{mg} / \mathrm{dl}$ with $5.1 \%$ IgG. Electromyography, motor nerve conduction velocity, and $\mathrm{F}$ waves were all normal. She displayed no clinical or 
electrophysiological evidence of motor nerve involvement. Sensory nerve conduction velocity was not detected in ulnar and tibial nerves.

The chronic onset of illness associated with sensory neuropathy and the axillar lymphadenopathy in this patient were suggestive of sensory neuropathy with malignancy of unknown origin. A biopsy of the left axillary lymph node disclosed metastatic adenocarcinoma. Repeated examinations failed to disclose the primary adenocarcinoma lesion. Immunohistological study using antithyroglobulin antibody, antiamyloid antibody, anti-CA19-9 antibody, anti-CA15-3(DF3, 115D8), and BCA225 suggested that metastatic adenocarcinoma of the left axillary lymph node originated from a breast cancer. On 16 December 1994, she underwent left radical mastectomy. Histological examination of a surgically obtained specimen showed intraductal spreading type breast cancer. Sural nerve biopsy was performed at the time of mastectomy, and showed pronounced axonal degeneration but no neoplastic cell infiltration. The patient was discharged in good health in January 1995, but sensory neuropathy remained. After surgery, her sensory neuropathy did not progress.

\section{Methods}

IMMUNOHISTOCHEMICAL IDENTIFICATION OF ANTINEURAL ANTIBODIES IN THE PATIENT'S SERUM.

Immunohistochemical studies were performed on frozen acetone fixed tissue sections using immunoperoxidase staining. Under deep anaesthesia, a mouse was perfused and the brain and spinal cord were removed. Frozen sections $4 \mu \mathrm{m}$ thick were prepared by cryostat from coronal slices of cerebellum and spinal cord from the mouse, and from the patient's left axillar lymph node. The sections were incubated with the serum from the patient or a normal healthy subject, diluted $1: 2000$ in $1 \%$ blotto (non-fat dry milk), for two hours at room temperature. After washing with phosphate buffered saline (PBS) the sections were incubated with biotinilated goat antihuman IgG antibody (KPL Inc), diluted 1:400 in 1\% blotto, for two hours at room temperature. After washing with PBS, they were reacted by an ABC method using histostain-DS kit (Zymed Lab Inc, San Francisco, CA, USA).

WESTERN BLOTTING ANALYSIS OF ANTINEURAL ANTIBODIES IN THE PATIENT'S SERUM

The mouse cerebellum and patient's left axillar lymph node were homogenised in PBS. The homogenate was mixed with $2 \%$ sodium dodecyl sulphate (SDS) $-5 \%$ 2-mercaptoethanol and separated by $12 \%$ polyacrylamide gel electrophoresis according to Laemmeli's method ${ }^{9}$ with a vertical slab gel apparatus. The proteins in the gel were transferred on to nitrocellulose membrane in tris-glycine-methanol buffer $(\mathrm{pH} 8.3)$ with an electroblotting apparatus (Bio-Rad). The membrane was preincubated with $1 \%$ blotto, and subsequently incubated with the serum of the patient, diluted 1:200 in $1 \%$ blotto, for two hours at room temperature. The membrane was washed with PBS and incubated with phosphatase conjugated goat antihuman IgG antibody (KPL Inc), diluted $1: 1000$ in $1 \%$ blotto, for two hours at room temperature. The membrane was then washed with PBS again, the colour reaction of alkaline phosphatase was developed by adding a mixture of nitroblue tetrazolium and 5-bromo-4-chloro-3-indol phosphate (Gibco BRL). We used the serum of a patient with limbic encephalitis and small cell carcioma with confirmed anti-Hu antibody in the serum for a positive control, and a normal healthy subject for a negative control.

\section{Results}

IMMUNOHISTOLOGICAL IDENTIFICATION OF ANTINEURAL ANTIBODY

Immunohistochemistry analysis of serum samples from the patient showed staining of the molecular layer and Purkinje cell layer of the cerebellar cortex as detected by light microscopy. In the spinal cord, the posterior grey horn was stained (figure 1). These findings could not be detected in samples from the normal control subject.

WESTERN BLOT ANALYSIS

The IgG antibodies against a $106 \mathrm{kDa}$ antigen in mouse cerebellum were detected in the serum of the patient (figure 2). Conversely, serum samples from the control did not react. Anti-Hu antibody was not detected in the serum of the patient or the control. The patient's serum did not react with her metastatic lymph node. Normal control serum did not react with the $106 \mathrm{kDa}$ protein in either cerebellum or lymph node. We also performed western blot analysis using mouse liver, lung, kidney, and skeletal muscle. No $106 \mathrm{kDa}$ antigen was found.

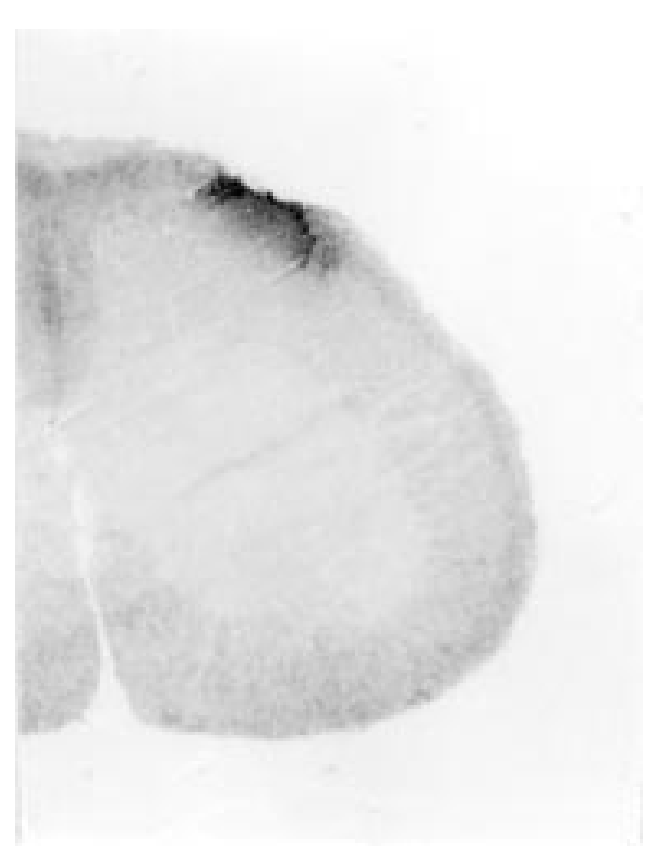

Figure 1 Immunohistochemical staining of frozen sectioned mouse spinal cord. Serum samples were diluted 1:2000 in 1\% blotto. The posterior grey horn of the spinal cord was stained. 


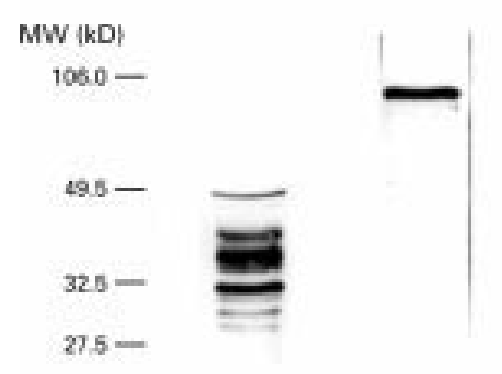

A.

B

Figure 2 Western blot analysis of $(A)$ patient with anti-Hu antibody positive limbic encephalitis with small cell lung cancer; (B) our patient; (C) normal control. The IgG antibodies against $106 \mathrm{kDa}$ antigen in mouse cerebellum were detected in the serum of the patient. Conversely, serum samples from the control did not react.

\section{Discussion}

The most common cancer with paraneoplastic sensory neuropathy is small cell lung cancer, and it is associated with autoantibodies against the $\mathrm{Hu}$ antigen in neuronal nuclei. Some cases of paraneoplastic sensory neuropathy with breast cancer have also been reported. ${ }^{1{ }^{10}}{ }^{11}$ Our case ran a chronic clinical course of asymmetric sensory neuropathy with breast carcinoma. After surgery, her neurological symptoms did not progress. It is suggested that her sensory neuropathy may be associated with the existence of carcinoma.

Although the precise cause and pathogenesis of paraneoplastic sensory neuropathy are not clarified, there is increasing evidence that paraneoplastic sensory neuropathy may be caused by immune mediated mechanisms. Autoantibodies have been detected in the serum of many patients, with various paraneoplastic neurological diseases, using immunohistochemistry and immunoblotting. ${ }^{12-15}$ In neurological paraneoplastic syndrome, several neural antibodies have been identified. ${ }^{16}$ AntiPurkinje cell autoantibody, so-called "Yo" antibody, has been a marker of gynaecological and breast carcinoma in the context of subacute cerebellar degeneration. Antineuronal nuclear antibodies, which are called "Hu" and "Ri", were found in patients with small cell lung cancer and associated sensory neuropathy and encephalomyeloradiculopathies. Paraneoplastic sensory neuropathy is often associated with anti-Hu antibody and small cell lung cancer. ${ }^{14-6}$

Although already known antineural antibodies associated with neurological paraneoplastic syndrome, such as anti-Hu, $\mathrm{Ri}$, or Yo antibody, were negative in our patient, we found an IgG antibody that reacted against mouse neural tissue in the patient's serum. Immunohistochemical study disclosed that IgG antibody was reacting with the molecular layer and Purkinje layer of cerebellum and dorsal roots and posterior grey horn of the spinal cord. Western blot analysis showed that an antibody was directed against a $106 \mathrm{kDa}$ mouse neural protein. It is very difficult to prove that the antibody that gives the $106 \mathrm{kDa}$ band in immunoblots of mouse cerebellum is the same that gives immunostaining in cerebellum or spinal cord. However, there is a possibility that the antibody that gives the $106 \mathrm{kDa}$ band in immunoblotted mouse neural tissue may be the same as that which gives immunostaining in cerebellum or spinal cord, although there is also a possibility that additional antibodies against conformational epitopes would have been missed by immunoblotting and be the ones that immunoreact with cerebellum and spinal cord.

Characteristic features of the pathology of paraneoplastic sensory neuropathy are degeneration of the dorsal root ganglion neurons and mononuclear infiltration of the dorsal root ganglia. Secondary Wallerian degeneration of peripheral sensory nerves, posterior nerve roots, and posterior columns of the spinal cord is evident. ${ }^{1{ }^{1011} 17}$ Although the relation between the antibody we described and sensory neuropathy is not clear, immunohistochemical study disclosed that this antibody seems to be related to paraneoplastic sensory neuropathy and may play an important part in the pathogenesis of this neuropathy.

The role of antineural antibodies in the pathogenesis of paraneoplastic sensory neuropathy is unknown. Some reports assert that paraneoplastic sensory neuropathy results from an autoimmune cross reaction between a tumour cell antigen and brain nucleoprotein. ${ }^{5}$ On the other hand, antibodies against neural tissue are also detected in the serum of normal subjects. ${ }^{18}{ }^{19}$ Sillevis et al reported that immunisation of the recombinant $\mathrm{Hu}$ fusion protein did not cause neurological disease in mice even though they had a high titre of anti-Hu antibodies. ${ }^{20}$ Taken together, these findings suggest that antibodies which cross react with a tumour cell and neural tissues do not directly result in the destruction and degeneration of the neural tissues. In this study, we have shown an antineural antibody directed against a 106 $\mathrm{kDa}$ neural protein that does not react with the metastatic lymph node. Our results do not support the hypothesis that the paraneoplastic sensory neuropathy of the patients is directly caused by an antibody that cross reacts with a tumour cell antigen and a neural antigen. However, this antibody may play an important part in the pathogenesis of neurological paraneoplastic syndrome. The role of antineural antibody should be clarified. In so far as we are aware, this is the first report of this antineural antibody in the serum of a patient with breast cancer and sensory neuropathy. It is possible that the antibody that we detected is a new antineural antibody. Further study is needed to clarify the specificity and sensitivity of this antibody to paraneoplastic sensory neuropathy or breast carcinoma.

We thank Dr Koichiro Sakai, Department of Neurology, Kanazawa Medical College, for examinations of anti-Hu, Yo, and $\mathrm{Ri}$ antibodies in the patient's serum. 1 Horwich MS, Cho L, Porro RS, Posner JB. Subacute Neurol 1977;2:7-19. 
2 Dalmau J, Graus F, Resenblum MK, Posner JB. Anti-Huassociated paraneoplastic encephalomyelitis/sensory neuropathy: a

3 Chalk CH, Windebank AJ, Kimmel DW, McManis PG. The distinctive clinical features of paraneoplastic sensory neuropathy. Can $\mathcal{f}$ Neurol Sci 1992;19:346-51.

4 Graus F, Cordon-Card C, Posner JB. Neuronal antinuclear antibody in sensory neuropathy from lung cancer. Neurology 1985;35:538-43.

5 Graus F, Elkon KB, Cordon-Card C, Posner JB. Sensory neuropathy and small cell lung cancer. Antineuronal antibody also reacts with the tumor. Am $\mathcal{F}$ Med 1986;80:45-52.

6 Dalmau J, Furneaux HM, Gralla RJ, Kris MG, Posner JB. Detection of anti-Hu antibody in the serum of patients with small cell lung cancer-a quantitative western blot analysis. Ann Neurol 1990;27:544-52.

7 Kiers L, Altermatt HJ, Lennon VA. Paraneoplastic antineuronal nuclear IgG autoantibodies (type I) localize antigen in small cell lung carcinoma. Mayo Clin Proc 1991;66: gen in small

8 Kimmel DW, O’Neill BP, Lennon VA. Subacute sensory neuropathy associated with small cell lung carcinoma: diagnosis aided by autoimmune serology. Mayo Clin Proc 1988;63:29-32.

9 Laemmeli UK. Cleavage of structural proteins during the assembly of the head bacteriophage T4. Nature 1970;227: $680-5$

10 Croft PB, Urich H, Wilkinson M. Peripheral neuropathy of the sensorimotor type associated with malignant disease. Brain 1967;90:31-66.

11 Henson RA, Russell DS, Wilkinson M. Carcinomatous neuropathy: a clinical and pathological study. Brain $1954 ; 77: 82-121$
12 O'Neill JH, Murray NM, Newsom-Davis J. The LambertEaton myasthenic syndrome. A review of 50 cases. Brain Eaton myasthenic

13 Folli F, Solimena M, Cofiel R, Austoni M, Tallini G, Fassetta $\mathrm{G}$, et al. Autoantibodies to a $128-\mathrm{kD}$ protein in three women with the stiff-man syndrome and breast cancer. N Engl f Med 1993;328:546-51.

14 Kelly JJ. Peripheral neuropathies associated with monoclonal proteins. A clinical review. Muscle Nerve 1985;8: 138-50.

15 Sher E, Gotti C, Canal N, Scoppetta C, Piccolo G, Evoli A, Clementi F. Specificity of calcium channel autoantibodies in Lambert-Eaton myasthenic syndrome. Lancet 1989;ii: in Lam $640-3$.

16 Lennon VA. Paraneoplastic autoantibodies: The case for a descriptive genergic nomenclature. Neurology 1994;44: 2236-40.

17 Anderson NE, Cunningham JM, Posner JB. Autoimmune pathogenesis of paraneoplastic neurological syndromes. CRC Neurobiology 1987;3:245-99.

18 Stefansson K, Marton LS, Diepperink ME. Circulating autoantibodies to the 200,000 -dalton protein of neurofilaments in the serum of healthy individuals. Science ments in the serun

19 Daar AS, Fabre JW. Organ-specific IgM autoantibodies to liver, heart and brain in man: generalized occurrence and possible functional significance in normal individuals, and studies in patients with multiple sclerosis. Clin Exp Immunol 1981;45:37-47.

20 Sillevis Smitt PAE, Manley GT, Posner JB. Immunization with the paraneoplastic encephalomyelitis antigen $\mathrm{HuD}$ does not cause neurologic disease in mice. Neurology 1995; 45:1873-8. 